\title{
Radiotracer localization of nonpalpable pulmonary nodules: A single-center experience
}

Sandra L. Starnes, MD, ${ }^{\mathrm{a}}$ Michael Wolujewicz, MD, ${ }^{\mathrm{b}}$ Julian Guitron, MD, ${ }^{\mathrm{a}}$ Valerie Williams, MD, ${ }^{\mathrm{a}}$

Jennifer Scheler, MD, ${ }^{\mathrm{c}}$ and Ross Ristagno, $\mathrm{MD}^{\mathrm{b}}$

\section{ABSTRACT}

Objective: Multiple localization techniques to facilitate intraoperative identification of small or nonsolid pulmonary nodules have been developed. Radiotracer localization using technetium-labeled macroaggregated albumin has been our preferred localization method since 2009. We report our experience, including technical pitfalls and modifications, of our initial 77 patients who underwent this technique.

Methods: All patients undergoing preoperative radiotracer localization were identified from a prospective database. Medical records were retrospectively reviewed for patient demographic characteristics, nodule characteristics, procedure details, pathologic data, and outcomes.

Results: Seventy-seven patients underwent localization of 79 pulmonary nodules. Radiotracer localization had an overall success rate of 95\%; however, 2 patients required a second localization procedure on the same day. Most failures occurred in nodules that were $<5 \mathrm{~mm}$ from the pleural surface, resulting in pleural spillage. Seventy-three patients underwent a diagnostic wedge resection, with 2 of these patients requiring 2 wedge resections. In 2 patients, the nodules were successfully localized; however, they were too deep for wedge resection and required anatomic resection. Two patients did not undergo resection. One patient developed pleural spillage and hemothorax and due to subsequent comorbidities, was never rescheduled. The second patient did not tolerate single-lung ventilation. The majority $(86 \%)$ of lesions were malignant. Median length of stay was 2 days (range, 1-15 days). There was no 30-day mortality. The only morbidity was a prolonged air leak ( $>5$ days) in 5 patients.

Conclusions: Radiotracer localization is a simple and effective technique for intraoperative identification of small pulmonary nodules. (J Thorac Cardiovasc Surg 2018;156:1986-92)

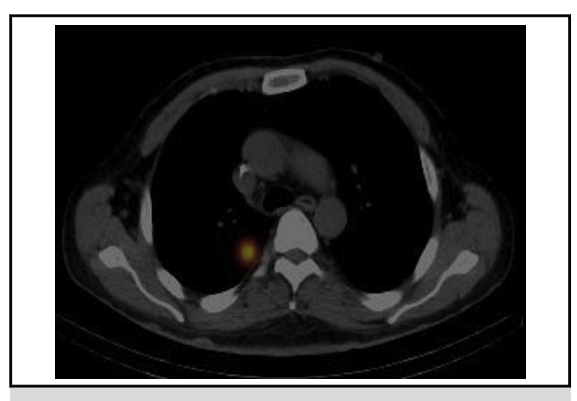

A dominant focus of uptake is seen on postradiotracer injection SPECT imaging.

\section{Central Message}

Radiotracer localization is a simple and effective technique for intraoperative localization of small or nonsolid pulmonary nodules.

\section{Perspective}

Multiple localization techniques to facilitate intraoperative identification of small or nonsolid pulmonary nodules have been developed, each with advantages and disadvantages. We report the outcomes, including technical pitfalls and modifications, of our initial experience with radiotracer localization. We found this technique to be simple and effective.

See Editorial Commentary page 1993.

See Editorial page 1972.
Thoracoscopic wedge resection is a well-establish method to diagnose pulmonary nodules, often done to confirm malignancy immediately before a planned anatomic resection. However, certain lesions can be difficult or impossible to

From the ${ }^{\mathrm{a}}$ Division of Thoracic Surgery, Department of Surgery, ${ }^{\mathrm{b}}$ Division of Interventional Radiology, Department of Radiology, and ${ }^{\mathrm{c}}$ Division of Nuclear Medicine, Department of Radiology, University of Cincinnati College of Medicine, Cincinnati, Ohio.

Received for publication Dec 6, 2017; revisions received March 5, 2018; accepted for publication March 5, 2018; available ahead of print May 16, 2018.

Address for reprints: Sandra L. Starnes, MD, Department of Surgery, University of Cincinnati College of Medicine, 231 Albert B Sabin Way, MC 0558, Cincinnati, OH 45267 (E-mail: sandra.starnes@uc.edu).

$0022-5223 / \$ 36.00$

Copyright $₫ 2018$ Published by Elsevier Inc. on behalf of The American Association for Thoracic Surgery

https://doi.org/10.1016/j.jtcvs.2018.03.152 identify with thoracoscopy, including those that are smaller than $1 \mathrm{~cm}$, have a depth from the pleural surface that is larger than their maximal diameter, have a part-solid consistency, or occur in the setting of reoperative resection. ${ }^{1}$ These same characteristics also make alternative biopsy methods, such as computed tomography (CT)-guided fineneedle aspiration or bronchoscopic biopsy challenging. With the increasing use of low-dose CT for lung cancer screening, an increasing number of small or part-solid

Scanning this QR code will take you to a supplemental video for the article.

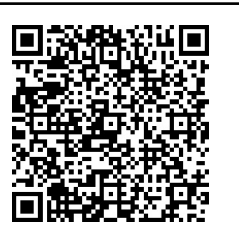




\section{Abbreviations and Acronyms \\ CT = computed tomography \\ RTL = radiotracer localization \\ SPECT/CT $=$ Single-photon emission computed tomography \\ Tc-99m MAA $=$ technetium-labeled macroaggregated albumin}

nodules have been identified with many of them ultimately requiring intervention.

To increase the success of a thoracoscopic wedge resection of nonpalpable pulmonary nodules, multiple localization techniques have been developed over the past couple of decades. These include preoperative percutaneous localization with methylene blue dye, ${ }^{2}$ hookwires or microcoils, ${ }^{3}$ intraoperative localization with bronchoscopic placement of dye or microcoils, ${ }^{4}$ and intraoperative ultrasound localization, among others. Each method has advantages and disadvantages. Radiotracer localization (RTL), in which a radioactive material is percutaneously injected in or near the pulmonary nodule and a radioprobe is subsequently used for intraoperative localization, was first introduced in 2000. Daniel's group at the University of Virginia ${ }^{6,7}$ modified the technique with the use of technetium-labeled macroaggregated albumin (Tc-99m MAA). Tc-99m MAA remains well localized where injected, has a half-life of 6 hours, and has abundant availability, making it an ideal radiotracer.

In 2009, we adopted Daniel's method of RTL. Here, we report our first 77 patients who underwent RTL of small or nonsolid pulmonary nodules before surgical resection. Technical details, imaging features, scintigraphy patterns, and outcomes are reviewed. RTL has seen limited use in a few centers in Europe and used infrequently in North America despite several advantages. Improved understanding of the application and potential technical pitfalls should facilitate greater application of this technique.

\section{METHODS \\ Patients}

All patients undergoing preoperative RTL between January 2009 and November 2016 at the University of Cincinnati Medical Center were identified from our prospective database. This encompasses the authors' entire experience with RTL, starting with the first patient. Medical records were retrospectively reviewed for patient demographic characteristics, nodule characteristics, preoperative evaluation, localization procedure, operative data, pathologic data, and postoperative morbidity and mortality. The study was approved by the University of Cincinnati Institutional Review Board and the need for informed consent was waived.

One of 4 dedicated general thoracic surgeons determined the need for RTL when difficult or impossible intraoperative thoracoscopic localization was anticipated. Guidelines for RTL used by all surgeons included nonsolid or part-solid opacities and nodules smaller in diameter than the distance to the pleural surface. We typically did not use preoperative localization for nodules that were believed to be amenable to blind anatomic resection based on location, such as in the apex of the lung. Contraindications to localization included a nodule adjacent to a giant bullae or a nodule that was inaccessible to percutaneous access.

\section{Localization Procedure}

All RTL procedures were performed by 1 board-certified interventional radiologist. The localization was performed using limited axial CT images (GE Light Speed QX/i, GE Healthcare, Piscataway, NJ) to determine the optimal approach to the lung nodule. Intravenous sedation and local anesthesia were utilized. A $10.9 \mathrm{~cm}, 19 \mathrm{G}$ coaxial needle was advanced toward the pulmonary opacity. A $15 \mathrm{~cm}, 22 \mathrm{G}$ Chiba needle was then inserted through the coaxial needle and the tip of the Chiba needle extended $1.5 \mathrm{~cm}$ beyond the tip of the coaxial needle. The radiotracer, approximately 0.2 to 0.3 millicurie (mCi) Tc-99m MAA (Draximage MAA, Kirkland, Quebec, Canada) in $0.2 \mathrm{~mL}$ was injected into or adjacent to the nodule. If the nodule was close to the pleura or major fissure, an effort was made to approach the lesion from the deep side of the lesion, thereby avoiding short intraparenchymal needle trajectories that might contribute to pleural spillage. Then, 0.1 to $0.2 \mathrm{~mL}$ of $1 \%$ lidocaine was injected through the Chiba needle to flush through the radiopharmaceutical agent. The Chiba needle was withdrawn into the coaxial needle and both needles were removed simultaneously. Any procedure-related material contaminated with Tc-99m MAA (eg, gloves, needles, syringes, sponges, and drapes) was isolated and allowed to decay in designated storage before medical waste disposal. The first 2 cases in our series involved concurrent hookwire localization. As we became comfortable with the RTL procedure, backup hookwire localization was not necessary.

If postprocedure CT images revealed a moderate $(>30 \%)$ or larger pneumothorax, $a>20 \%$ pneumothorax in patients with underlying emphysema, or a symptomatic pneumothorax, a small-caliber chest tube was inserted while the patient was still in the CT suite. After injection of the radiotracer, planar scintigraphy with frontal and lateral images (Siemens e.cam; Siemens Medical Solutions, Malvern, Pa) was performed. Singlephoton emission CT (SPECT/CT) (Symbia T6 SPECT/CT; Siemens, Munich, Germany) was performed only if pleural radiotracer contamination was suspected or if there were multiple foci of uptake of unclear location. If a focus of uptake was present within the approximate region of injected radiotracer and there was no pleural contamination, the patient proceeded to surgery. The interventional radiologist directly communicated the scintigraphy results and the relationship of injected radiotracer with the pulmonary opacity to the thoracic surgeon to complement the images that were available to the surgeon in the operating room.

RTL primary success was defined as radiotracer-identified in the lung at the site of the nodule of interest after 1 radiotracer injection. Secondary success was defined as radiotracer identified in the lung at the site of the nodule of interest after 1 or 2 attempts at radiotracer injection on the day of the intended procedure.

\section{Surgical Procedure}

Following successful localization, patients were transported to the operating suite and underwent wedge resection for diagnosis the same day. All procedures were initiated as video-assisted thoracoscopic surgery using 3 $12-\mathrm{mm}$ ports. The nodule was localized using an intraoperative gamma probe (RMD Instruments, Watertown, Mass) and wedge resection of the area identified was undertaken (Video 1). Frozen section was performed and formal lobectomy was undertaken at the same operation if invasive non-small cell lung cancer was identified and anatomic resection was planned.

\section{RESULTS}

From January 2009 to September 2016, 77 patients underwent RTL for 79 lung nodules. Two patients underwent 


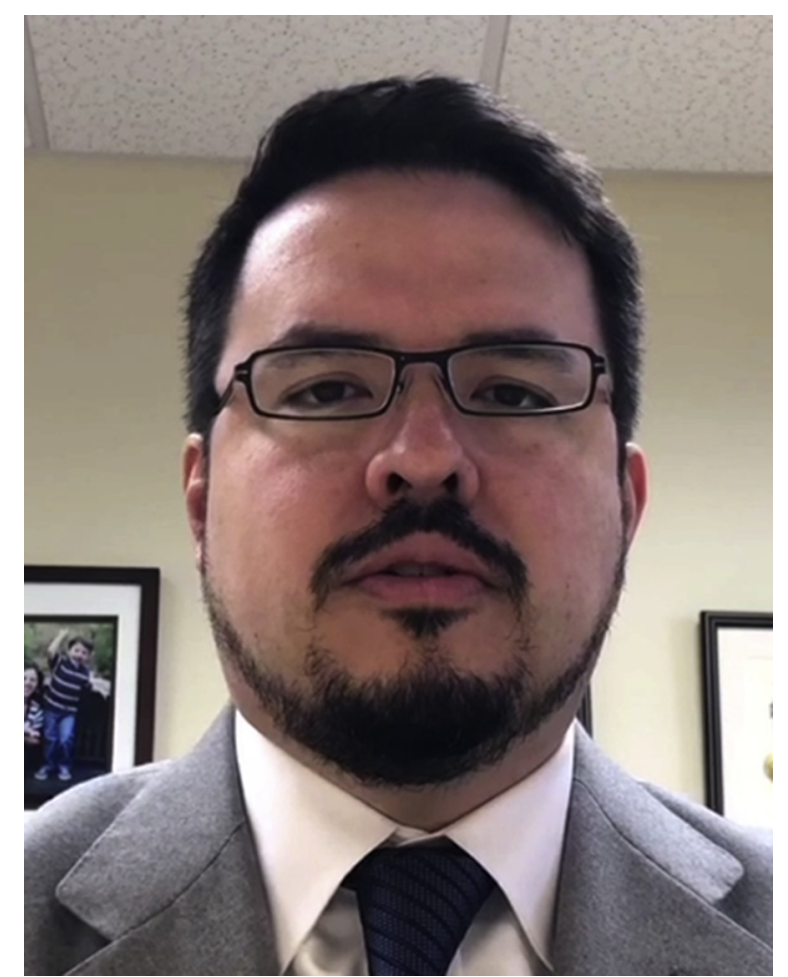

VIDEO 1. A description of the intraoperative technique for identification of nodules using radiotracer localization. Video available at: https://www. jtcvs.org/article/S0022-5223(18)30945-0/fulltext.

localization of 2 nodules on the same day. Patient demographic characteristics and nodule characteristics are outlined in Table 1. Forty-six patients $(60 \%)$ had a history of a current or previous cancer. Seventeen patients $(22 \%)$ had a prior lung cancer and $21(27 \%)$ had prior lung surgery, including 16 lobectomies and 5 wedge resections. The median nodule size was $10 \mathrm{~mm}$ (range, 2-33 mm) and $56 \%$ were ground glass opacities or part-solid nodules. Solid nodules tended to be smaller than pure ground glass or part-solid nodules with median size of $7 \mathrm{~mm}$ (range, 2$11 \mathrm{~mm}$ ) versus $14 \mathrm{~mm}$ (range, 4-32 mm). The most common indications for surgery included nodule growth $(53 \%)$, suspected metastatic disease $(23 \%)$, or development or growth of a solid component in a part solid nodule $(10 \%)$. Figure 1 shows a representative CT image from a patient with an enlarging ground glass opacity nodule.

\section{RTL}

Procedural characteristics are outlined in Table 2. RTL had a primary success rate of $92.4 \%$ (73 out of 79) a secondary success rate of $95 \%$ ( 75 out of 79 ). Two patients required a second localization procedure on the same day. In the first patient (patient \#6), no tracer was seen on the scintigram and he underwent an immediate repeat localization, which was successful. In another patient (patient \#55), the radiotracer was seen in the major fissure on the scintigram and he also underwent a repeat localization the
TABLE 1. Patient demographic characteristics and nodule characteristics

\begin{tabular}{|c|c|}
\hline Characteristic & Result \\
\hline Patients & 77 \\
\hline Nodules* & 79 \\
\hline Age, $y$ & $62(40-83)$ \\
\hline \multicolumn{2}{|l|}{ Gender } \\
\hline Male & $20(26)$ \\
\hline Female & $57(74)$ \\
\hline \multicolumn{2}{|l|}{ Race } \\
\hline White & $64(83)$ \\
\hline African American & $12(16)$ \\
\hline Asian & $1(1)$ \\
\hline Smoking history & $61(79)$ \\
\hline Pack-years & $41 \pm 24$ \\
\hline Forced expiratory volume in $1 \mathrm{~s}, \%$ & $83 \pm 23$ \\
\hline Diffusion capacity for carbon monoxide, $\%$ & $79 \pm 22$ \\
\hline \multicolumn{2}{|l|}{ Chronic obstructive pulmonary disease $\dagger$} \\
\hline Yes & $25(32)$ \\
\hline No & $23(30)$ \\
\hline Unknown & $29(38)$ \\
\hline Nodule size, $\mathrm{mm}$ & $10(2-33)$ \\
\hline \multicolumn{2}{|l|}{ Lobe } \\
\hline Right upper lobe & $24(30)$ \\
\hline Right middle lobe & $6(7)$ \\
\hline Right lower lobe & $14(18)$ \\
\hline Left upper lobe & $25(32)$ \\
\hline Left lower lobe & $10(13)$ \\
\hline \multicolumn{2}{|l|}{ Nodule characteristic } \\
\hline Solid & $35(44)$ \\
\hline Part solid & $28(36)$ \\
\hline Ground glass opacity & $16(20)$ \\
\hline Distance to pleura, $\mathrm{mm}$ & $5(0-24)$ \\
\hline
\end{tabular}

Values are presented as $\mathrm{n}$, median (range), $\mathrm{n}(\%)$, or mean \pm standard deviation. *Two patients had 2 nodules localized at the same time. $\dagger$ Based on Global Initiative for Chronic Obstructive Lung Disease definition.

same day, which was successful. Four patients failed localization. Three patients had diffuse pleural contamination on scintigraphy. All 3 had nodules that were $\leq 4 \mathrm{~mm}$ from the pleural surface. One patient underwent a successful hookwire localization 2 days later. One patient developed a hemothorax, requiring chest tube drainage. Due to declining functional status from his comorbidities, the nodule was followed with surveillance CT. The third patient underwent the scheduled surgical procedure the same day and the nodule was successfully removed based on anatomic location. A fourth patient appeared to have a successful localization on scintigram; however, at the time of surgery, the radiotracer was found in the pericardial fat pad. This nodule was $6 \mathrm{~mm}$ from the pleural surface. She underwent successful wedge resection of the lingular lesion based on anatomic location on CT imaging. Two patients underwent RTL of 2 


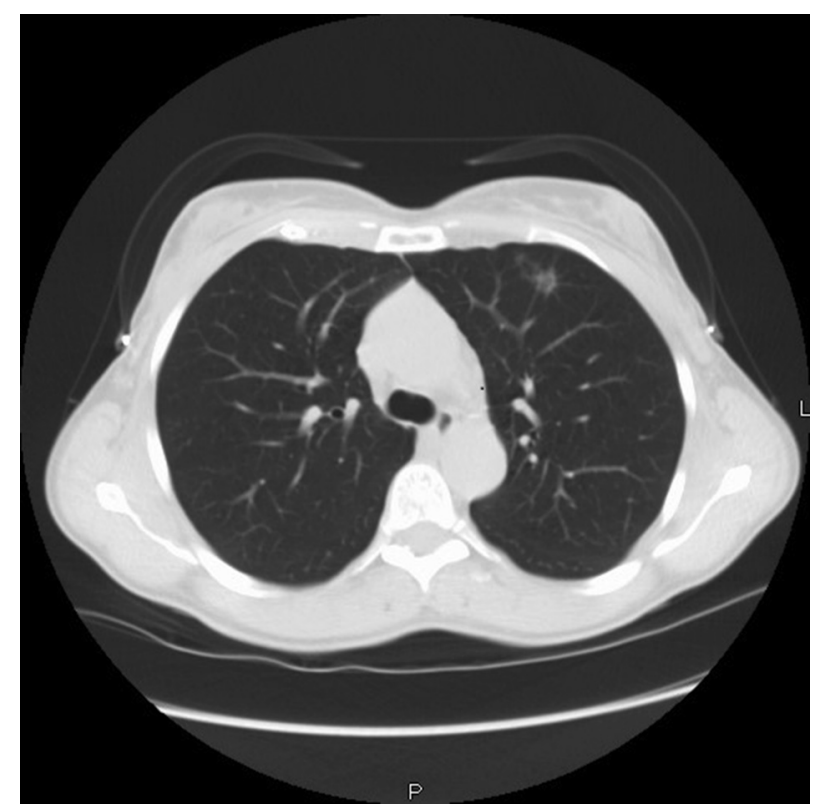

FIGURE 1. Representative computed tomography image from a patient with an enlarging ground glass opacity.

pulmonary nodules in the same lobe. In the first case, the nodules were $9.55 \mathrm{~cm}$ apart and the radiotracer activity $(0.313$ and $0.316 \mathrm{mCi})$ resulted in overlapping radiation fields, which slightly prolonged the intraoperative localization. For the next patient with RTL of 2 nodules $(9.76 \mathrm{~cm}$ apart), a lower activity of Tc-99m MAA (0.175 and $0.195 \mathrm{mCi}$ ) was used, which allowed for easier localization of the 2 nodules.

Ninety percent of patients had no complications from the localization procedure or a small asymptomatic pneumothorax that did not require intervention. A pneumothorax requiring placement of a small-bore chest tube occurred in $9 \%$ of patients and 1 patient developed a delayed hemothorax requiring tube thoracostomy as described above. Twenty percent of patients ( 5 out of 25 ) with documented chronic obstructive pulmonary disease developed a pneumothorax requiring a chest tube placement versus $0 \%(0$ out of 23) in patients without chronic obstructive pulmonary disease and $7 \%$ of patients ( 2 out of 29 ) in which a chronic obstructive pulmonary disease diagnosis was unknown due to lack of pulmonary function testing. None of the patients who developed a pneumothorax had significant bullous disease because this was a contraindication to RTL.

\section{Scintigraphy}

All patients underwent nuclear scintigraphy following RTL. Planar images were obtained in all patients but 2, in which only SPECT/CT images were obtained. SPECT/CT was performed in 9 patients. Five patterns of uptake were identified on scintigraphy: single focus of uptake, single dominant focus with indeterminate secondary focus/foci,
TABLE 2. Procedure characteristics and outcomes

\begin{tabular}{lc}
\hline \multicolumn{1}{c}{ Characteristic } & Outcome \\
\hline Technetium-labeled macroaggregated albumin, & $0.18(0.05-0.7)$ \\
millicuries & \\
Radiotracer localization success & $73(92.4)$ \\
Primary success & $75(95)$ \\
Secondary success & $4(5)$ \\
Failure & \\
Complications of radiotracer localization & $40(52)$ \\
None & $29(38)$ \\
Pneumothorax (no chest tube) & $7(9)$ \\
Pneumothorax (chest tube placed) & $1(1)$ \\
Hemothorax & \\
Scintigraphy findings & $46(58)$ \\
Single focus of uptake & $18(23)$ \\
Dominant focus plus small secondary & $7(9)$ \\
Dominant focus plus tail & $5(6)$ \\
Dominant focus plus airway penetration & $3(4)$ \\
Pleural spillage & \\
Operative procedures & $68(86)$ \\
Wedge resection & $6(8)$ \\
Wedge followed by lobectomy & $1(1)$ \\
Wedge followed by segmentectomy & $1(1)$ \\
Lobectomy & $1(1)$ \\
Segmentectomy & $2(3)$ \\
No resection & $112 \pm 70$ \\
Length of surgery, min & $50(65)$ \\
Pathologic diagnosis & $16(21)$ \\
Lung cancer & $11(14)$ \\
Metastasis & \\
Benign & \\
\hline & \\
\hline
\end{tabular}

Values are presented as median (range), $\mathrm{n}(\%)$, or mean \pm standard deviation.

single focus with tail, single focus with airway penetration with or without gastrointestinal extension, and pleural contamination. A single focus was seen in the majority $(58 \%)$, corresponding to focally deposited radiotracer (Figure 2, A). A secondary focus separate from and fainter than the primary site of uptake, usually in the hilum or mediastinum, was appreciated 23\% (Figure 2, B). Seven patients demonstrated a single focus with adjacent illdefined hazy uptake, termed a tail (Figure 2,C). Five patients demonstrated secondary foci of uptake characteristic of endobronchial spread secondary to airway penetration of the radiotracer (Figure 2, D). Some of these patients also demonstrated radiotracer in the gastrointestinal tract, anywhere from the pharynx to the stomach, due to swallowed radiotracer brought up from the bronchial tree. Anecdotally, penetration of radiotracer into the airway occurred with more vigorous injection of radiotracer. None of these imaging findings influenced intraoperative localization.

\section{Surgical Procedures and Outcomes}

Seventy-three patients underwent an initial wedge resection, with 2 of these patients each undergoing 2 wedge 

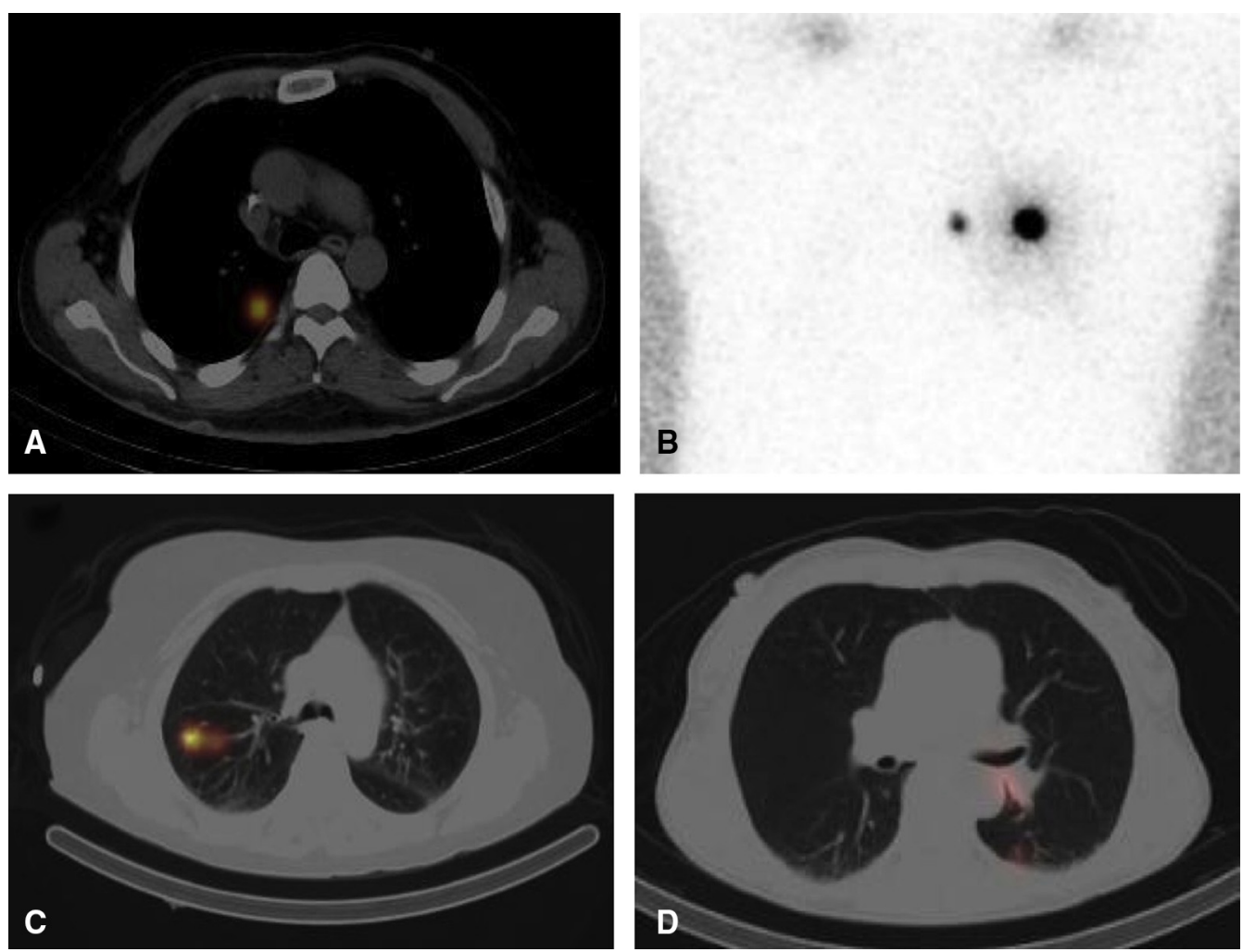

FIGURE 2. Patterns seen on postradiotracer injection scintigraphy. A, Single focus of uptake seen on single-photon emission computed tomography. B, Dominant focus with a smaller secondary focus seen on planar scintigraphy. C, Single dominant focus with an adjacent tail. D, Airway penetration seen on single-photon emission computed tomography.

resections, all performed thoracoscopically (Table 2). Anatomic resection with segmentectomy or lobectomy was performed in 7 of these patients. The remaining patients were treated with wedge resection alone due to benign pathology, resection for metastatic lesions, or lung cancers that were $<1 \mathrm{~cm}$ in size, inability to tolerate lobectomy, minimally invasive adenocarcinoma or adenocarcinoma in situ. In 2 patients, the nodules were successfully localized; however, they were too deep for a wedge resection. One of these patients underwent a segmentectomy and the other underwent lobectomy. Two patients did not undergo resection. In 1 patient, described previously, the procedure was aborted due to pleural spillage and hemothorax and due to subsequent comorbidities, he was never rescheduled. A second patient could not tolerate single-lung ventilation and the procedure was aborted.

The majority ( $86 \%$ ) of lesions were malignant (Table 2). Of 50 lung cancers, 29 were adenocarcinoma, 8 were adenocarcinoma in situ, 8 were minimally invasive adenocarcinoma, and 5 were squamous cell carcinoma. Thirty-nine of 42 invasive malignancies $(93 \%)$ were stage IA, with 2 being stage IB and 1 being stage IIA. Of the patients undergoing curative intent resection, all had an R0 resection except 1 patient who had adenocarcinoma in situ at the margin and was not a candidate for further resection. The median margin was $15 \mathrm{~mm}$ (range, 3-50 mm).

Median length of stay was 2 days (range 1-15 days). There was no 30-day or in-hospital mortality. The only morbidity was a prolonged air leak ( $>5$ days) in 5 patients.

\section{DISCUSSION}

Thoracic surgeons are increasingly evaluating patients with small or nonsolid pulmonary nodules, especially in light of the increasing use of low-dose CT lung cancer screening. Nodules that are $<1 \mathrm{~cm}$ in size, nonsolid, or part solid, or are smaller in size than the distance to the pleural surface are difficult or impossible to identify intraoperatively with thoracoscopy, often necessitating long operative times, large blind wedge resections, or conversion to thoracotomy. ${ }^{1}$ Thus, we have seen the rising need for localization procedures. There is no universally preferred method and each method has advantages and disadvantages. Hookwire localization, one of the most commonly employed localization techniques used in the United States, is prone to wire dislodgement before and during surgery. The reported success rates range from $68 \%$ to $100 \%{ }^{8}{ }^{8}$ Complications include pneumothorax with reported rates 
up to $68 \%$, parenchymal hemorrhage, and air embolism. ${ }^{2,3,8,9}$ In addition, the surgeon must follow the trajectory of the hookwire pathway, which may result in a larger wedge resection. CT or bronchoscopy-guided injection of methylene blue dye is a technique used by many centers. ${ }^{4,10}$ This is a simple technique; however, diffusion over the pleural surface may make localization difficult and this method does not mark the depth of the lesion. CT-guided injection of a platinum microcoil typically involves technically difficult positioning of the outer tail of the coil into the pleural space and the use of intraoperative fluoroscopy, which can be cumbersome in the lateral position. ${ }^{11}$ Intraoperative ultrasound has the advantage of not requiring a preoperative procedure; however, it is operator-dependent and is less useful in patients with significant emphysema or in nonsolid nodules.

RTL was first described by Chella and colleagues in $2000^{5}$ using a radiotracer not available in the United States. Daniel's group ${ }^{6}$ studied several radiotracers and chose Tc-99m MAA as ideal for localization because the larger particle size minimizes diffusion and allows flexibility in surgical scheduling. This technique has several advantages. The material is easily available because it is used in lung perfusion scintigraphy. Once in place, dislodgement or diffusion does not occur. It enables the determination of nodule depth, which is not possible with many of the other techniques, and allows the surgeon to use the best orientation for wedge resection. Reported success rates range from $95 \%$ to $99 \%$. $^{7,12-16}$ One randomized trial demonstrated a success of $96 \%$ using RTL versus $84 \%$ with hookwire localization. ${ }^{12}$

At our center, we initially used CT-guided hookwire placement for localization of small pulmonary nodules. However, we had several instances of dislodgement of the hookwire with failed localization. In January 2009, we began using RTL, applying the method described by Daniel and colleagues. ${ }^{6}$ During our initial experience, we added a hookwire localization until we were comfortable with the new technique. However, after only 2 patients, we abandoned the hookwire method and RTL has been our preferred technique ever since. We found it to be reliable with a $95 \%$ success rate and efficient, enabling us to localize nodules within seconds. We did have 4 localization failures. Three of these patients had pleural spillage. All of these nodules were within $4 \mathrm{~mm}$ of the pleural surface, which likely increased the risk of pleural contamination. One of these patients experienced a hemothorax. This patient refused to receive intravenous sedation and developed intense coughing during the procedure. Whereas our practice has been to postpone thoracic surgery in patients with pleural contamination, other centers manage pleural contamination with intraoperative lavage to reduce background radiation. ${ }^{13}$ The other failure had an apparent successful localization by scintigraphy; however, the radiotracer was identified in the pericardial fat pad at the time of surgery. This nodule, located in the lingula, was also close to the pleural surface. Because all of these failures occurred in nodules that were within $6 \mathrm{~mm}$ of the pleural surface, we have adjusted our technique to place the radiotracer deep to the pleural surface, ideally at least $1 \mathrm{~cm}$ in depth.

In our series, scintigraphy performed following RTL was a valuable diagnostic tool to confirm the presence of a dominant focus of radiotracer marking the pulmonary opacity before sending the patient to the operating room. One patient early in our series had no tracer identified on scintigram. Following this, we routinely added a 0.1 to $0.2 \mathrm{~mL}$ of $1 \%$ lidocaine flush following radiotracer injection to improve clearance through the needle and we have not encountered this problem since. We observed several scintigraphic patterns following RTL. In addition to the expected dominant focus of radiotracer corresponding to the deposition, fainter secondary foci were identified in nearly half the patients $(47 \%)$. None of these patterns influenced successful localization, likely due to the intensity of the radioactivity at the primary injection site. The precise location and cause for the secondary uptake was often unknown, and may be related to passage through airways or lymphatic ducts. Other centers, particularly in Europe, add 0.1 to $0.2 \mathrm{~mL}$ nonionic contrast medium to the radiotracer to confirm appropriate localization on the postinjection CT. ${ }^{14,15}$ In our experience, the CT scan after radiotracer injection adequately demonstrates the injection site as a focus of ground glass opacity. Although the location of the ground glass opacity or contrast is helpful, scintigraphy confirms adequate radiotracer deposition and the absence of pleural contamination before initiating video-assisted thoracoscopic surgery.

SPECT/CT was performed in 9 patients to elucidate the precise radiotracer location. Despite more precise anatomic information, additional SPECT/CT imaging provides no additional useful clinical information beyond planar imaging and contributes to delays in patient arrival to the operating room; therefore, we now only use planar scintigraphy unless there is a particular need for more precise imaging.

Based on our experience with RTL, this is now our preferred technique for localization of small and nonsolid pulmonary nodules. We found this technique to be easy to use and effective. The ability to determine the depth of the nodule is a strong advantage, allowing a surgeon to use the most appropriate plane for wedge resection, thus limiting the amount of lung removed. The specific radiotracer that was used is available at most institutions and the low dose avoids the need for specialized radiation precautions. ${ }^{6}$ There are some disadvantages to this technique. Unless the localization procedure is done the day before, the operative procedure is delayed until completion of the radiotracer injection and confirmatory planar scintigraphy. With increasing experience, we were able to decrease the time of the RTL. We typically scheduled these procedures as a 
second case, thereby optimizing operating room use. It may be possible to schedule the RTL the day before planned resection as described by Grogan and colleagues ${ }^{13}$; however, a slightly higher dose of Tc-99m MAA should be used. We do not have experience with this because we prefer to schedule the procedures on the same day for patient convenience. The RTL technique is accompanied by added equipment costs such as the intraoperative gamma probe, personnel costs for the interventional radiology procedure, and imaging costs for the postprocedure planar scintigraphy. Although Grogan and colleagues ${ }^{17}$ demonstrated in a decision analysis that RTL with thoracoscopy was more costeffective than thoracotomy for small pulmonary nodules, additional studies are needed to address cost-effective comparisons between different localization techniques.

We appreciate several limitations in this study. The sample size is small, retrospective, and from a single center; however, it does include our entire experience and is one of only a few series from the United States. Based on progressive experience, minor modifications to the RTL technique, as described above, were implemented to continue to refine the success of the technique. Because this was a retrospective analysis, we do not have exact measurement of the intraoperative time required for localization; however, based on the authors' observation, this generally took $<1$ minute.

\section{CONCLUSIONS}

RTL is a simple and effective technique to identify small or nonsolid pulmonary nodules and facilitates thoracoscopic wedge resections, avoiding unnecessary lobectomy or thoracotomy. This technique overcomes some of the disadvantages of other techniques. It is a simple technique to implement, requiring no additional expertise on the part of the thoracic surgeon or interventional radiologist. It allows for precise and fast localization, including nodule depth, allowing a surgeon to use the best trajectory for wedge resection. Once in place, displacement does not occur. To implement this technique, collaboration between the thoracic surgeon and interventional radiologist is necessary. We had several meetings before initiating RTL at our institution and have had ongoing communication and review of our results. This has allowed us to make minor adjustments in technique to continually improve our outcomes. Localization techniques for small pulmonary opacities will likely become more prevalent with the increase use of CT screening for lung cancer. RTL has many advantages over other techniques and can be performed with high success and low morbidity.

\section{Conflicts of Interest and Statement}

Authors have nothing to disclose with regard to commercial support.

\section{References}

1. Suzuki K, Nagai K, Yoshida J, Ohmatsu H, Takahashi K, Nishimura M, et al. Video-assisted thoracoscopic surgery for small indeterminate pulmonary nodules: indications for preoperative marking. Chest. 1999;115:563-8.

2. Kleedehn M, Kim DH, Lee FT, Lubner MG, Robbins JB, Ziemlewicz TJ, et al. Preoperative pulmonary nodule localization: a comparison of methylene blue and hookwire techniques. AJR Am J Roentgenol. 2016;207:1334-9.

3. Hanauer M, Perentes JY, Krueger T, Ris HB, Bize P, Schmidt S, et al. Pre-operative localization of solitary pulmonary nodules with computed tomographyguided hook wire: report of 181 patients. J Cardiothorac Surg. 2016;11:5-11.

4. Awais O, Reidy MR, Mehta K, Bianco V, Gooding WE, Schuchert MJ, et al. Electromagnetic navigation bronchoscopy-guided dye marking for thoracoscopic resection of pulmonary nodules. Ann Thorac Surg. 2016;102:223-9.

5. Chella A, Lucchi M, Ambrogi MC, Menconi G, Melfi FMA, Gonfiotti A, et al. A pilot study of the role of TC-99 radionuclide in localization of pulmonary nodular lesions for thoracoscopic resection. Eur J Cardiothorac Surg. 2000;18:17-21.

6. Daniel TM, Altes TA, Rehm PK, Williams MB, Jones DR, Stolin AV, et al. A novel technique for localization and excisional biopsy of small of ill-defined pulmonary lesions. Ann Thorac Surg. 2004;77:1756-62.

7. Stiles BM, Altes TA, Jones DR, Shen KR, Ailawadi G, Gay SB, et al. Clinical experience with radiotracer-guided thoracoscopic biopsy of small, indeterminate lung nodules. Ann Thorac Surg. 2006;82:1191-7.

8. Park CH, Han K, Hur J, Lee SM, Lee JW, Hwang SH, et al. Comparative effectiveness and safety of pre-operative lung localization for pulmonary nodules: a systematic review and meta-analysis. Chest. 2017;151:316-28.

9. Ichinose J, Kohno T, Fujimori S, Harano T, Suzuki S. Efficacy and complications of computed tomography-guided hook wire localization. Ann Thorac Surg. 2013; 96:1203-8.

10. Marino KA, Sullivan JL, Weksler B. Electromagnetic navigation bronchoscopy for identifying lung nodules for thoracoscopic resection. Ann Thorac Surg. 2016;102:454-7.

11. Finley RJ, Mayo JR, Grant K, Clifton JC, English J, Leo J, et al. Preoperative computed tomography-guided microcoil localization of small peripheral pulmonary nodules: a prospective randomized controlled trial. J Thorac Cardiovasc Surg. 2015;149:26-32.

12. Gonfiotti A, Davini F, Vaggelli L, De Francisci A, Caldarella A, Gigli PM, et al. Thoracoscopic localization techniques for patients with solitary pulmonary nodule: hook wire versus radio-guided surgery. Eur J Cardiothorac Surg. 2007;32:843-7.

13. Grogan EL, Jones DR, Kozower BD, Simmons WD, Daniel TM. Identification of small lung nodules: technique of radiotracer-guided thoracoscopic biopsy. Ann Thorac Surg. 2008;85:S772-7.

14. Bellomi M, Veronesi G, Trifiro G, Brambilla S, Bonello L, Preda L, et al. Computed tomography-guided preoperative radiotracer localization of nonpalpable lung nodules. Ann Thorac Surg. 2010;90:1759-65.

15. Ambrogi MC, Melfi F, Zirafa C, Lucchi M, De Liperi A, Mariani G, et al. Radioguided thoracoscopic surgery (RGTS) of small pulmonary nodules. Surg Endosc. 2012;26:914-9.

16. Galetta D, Bellomi M, Grana C, Spaggiari L. Radio-guided localization and resection of small or ill-defined pulmonary lesions. Ann Thorac Surg. 2015; 100:1175-80.

17. Grogan EL, Stukenborg GJ, Nagji AS, Simmons W, Kozower BD, Jones DR, et al. Radiotracer-guided thoracoscopic resection is a cost-effective technique for the evaluation of subcentimeter pulmonary nodules. Ann Thorac Surg. 2008;86:934-40.

Key Words: localization, radiotracer localization, pulmonary nodule 